\title{
Milestones in robotic colorectal surgery development: an historical overview
}

\author{
Pietro Genova', Gianni Pantuso', Solafah Abdalla², Riccardo Memeo ${ }^{3}$, Federica Gaiani ${ }^{4}$, Paschalis \\ Gavriilidis $^{5}$, Nicola de'Angelis ${ }^{6}$
}

\begin{abstract}
'Department of General and Oncological Surgery, Paolo Giaccone University Hospital, University of Palermo, 90127 Palermo (PA), Italy.

${ }^{2}$ Department of Digestive Surgery and Surgical Oncology, Bicêtre University Hospital, Assistance Publique Hôpitaux de Paris, Université Paris-Sud, 78 Rue du Général Leclerc, Le Kremlin Bicêtre 94275, France.

${ }^{3}$ Department of Emergency and Organ Transplantation, Institute of General Surgery and Liver Transplantation, University of Bari, 70124 Bari (BA) Italy.

${ }^{4}$ Gastroenterology and Endoscopy Unit, Department of Medicine and Surgery, University of Parma, PR Gastroenterology and Endoscopy Unit, Department of Medicine and Surgery, University of Parma, PR IT-43124, Italy.

${ }^{5}$ Department of Hepato-Pancreato-Biliary and Liver Transplant Surgery, Queen Elizabeth University Hospitals Birmingham NHS Foundation Trust, Birmingham B15 2TH, UK.

${ }^{6}$ Department of Digestive and Hepato-Pancreato-Biliary Surgery, Henri Mondor University Hospital, AP-HP, Université ParisEst Créteil (UPEC), 51 Avenue du Maréchal de Lattre de Tassigny, Créteil 94010, France.
\end{abstract} Correspondence to: Dr. Pietro Genova, Department of General and Oncological Surgery, Paolo Giaccone University Hospital,
University of Palermo, Via del Vespro 129, 90127 Palermo (PA), Italy. E-mail: pietro.genova@gmail.com

\begin{abstract}
How to cite this article: Genova P, Pantuso G, Abdalla S, Memeo R, Gaiani F, Gavriilidis P, de'Angelis N. Milestones in robotic colorectal surgery development: an historical overview. Mini-invasive Surg 2020;4:2.

http://dx.doi.org/10.20517/2574-1225.2019.30
\end{abstract}

Received: 29 Aug 2019 First Decision: 5 Nov 2019 Revised: 25 Nov 2019 Accepted: 10 Dec 2019 Published: 7 Jan 2020

Science Editor: Nicola de'Angelis Copy Editor: Cai-Hong Wang Production Editor: Tian Zhang

\begin{abstract}
The present article is a historical review intended to trace the most important phases in the development of robotic surgical technology, with a special focus on colorectal surgery. The initial section considers the origin and some etymological aspects of the word "robot". Then, a historical overview traces the development of robotic technology in industry and its implementation within the operating theatres. Finally, the first publications concerning robot-assisted colon and rectal surgery are reported together with a brief state of the art about this issue.
\end{abstract}

Keywords: Robotic surgery, colorectal surgery, history

\section{INTRODUCTION}

In the contemporary world, the surprising and fascinating development of digital electronic technologies has determined so many changes in all aspects of human life that we now speak of a "digital revolution". An

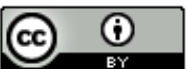

(C) The Author(s) 2020. Open Access This article is licensed under a Creative Commons Attribution 4.0 International License (https://creativecommons.org/licenses/by/4.0/), which permits unrestricted use, sharing, adaptation, distribution and reproduction in any medium or format, for any purpose, even commercially, as long as you give appropriate credit to the original author(s) and the source, provide a link to the Creative Commons license, and indicate if changes were made.

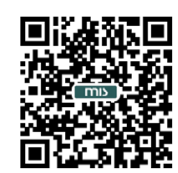


evident result of these impressive advances is certainly represented by the widespread use of robots. With this term, we refer to a large variety of machines with very different purposes and levels of complexity, from industrial manufacturing systems to humanoids provided with artificial intelligence. Over time, robotic technology has been naturally implemented also in the operating theatres, becoming one of the most debated topics in surgery over the last years. In the present article, we focus the observation field to general surgery, trying to trace the most important phases in the development of robotics in colorectal surgery.

\section{DEFINITION AND ETYMOLOGY}

The word "robot" is used to indicate a programmable machine able to carry out several tasks in aid of, or in place of, men with a variable degree of autonomy. It comes from the Czech noun "robota", for forced labor or hard work, reminding of the status of serfdom in the feudal society, and sharing the same radical of the words meaning "work" in many current Slavic languages, such as Polish, Ukrainian, and Russian ${ }^{[1]}$.

The Czech writer Karel Čapek first used this term in his science fiction play titled "R.U.R." (Rossum's Universal Robots), published in 1920. This work deals with artificial workers, indistinguishable from men, produced from synthetic organic matter with the purpose of freeing mankind from physical fatigue. However, "robots" rapidly spread all over, start rebelling against men, and conquer the world.

Later, in 1943, the visionary American writer Isaac Asimov first used the word "robotics" in his science fiction story titled "Runaround". Here, he established the three "Laws of Robotics", a set of rules hardwired into the artificial brain of autonomous humanoid robots to underlie their behavior and prevent them from rebelling against their creators.

Hence, since their origin, two main concepts converge into the terms "robot" and "robotics": work in support of men and the need of control by the latter.

\section{HISTORY}

Knowing the history of the implementation of robotic technology in surgery is useful for appreciating current advances in this field. Here, some important landmarks are reported to offer a general overview.

\section{Ancient times: "automata"}

From ancient times, the idea of self-operating machines, usually indicated as "automata", is present in all cultures, from west to east.

The word "automaton" (plural "automata") comes from the Greek word " $\alpha$ v่ meaning "something self-acting", which was first used by Homer in the Iliad referring to self-opening doors and self-moving wheeled tripods.

Several myths of the Greek and Roman tradition deal with "automata". In the Iliad, Homer tells that Hephaestus, the god of fire, forges, metallurgy, and sculpture, constructed golden handmaids to help him in his forge on the Island of Lemnos. In the "Metamorphoses", Ovid reports the myth of Pygmalion, a sculptor falling in love with his statue, Galatea, who was transformed into an animated creature by the goddess Aphrodite to fulfill Pygmalion's prayer.

Halfway around the world, in ancient China, during the Zhou Dynasty (1023-957 BC), the artificer Yan Shi showed to King Mu a human-like machine that could move and sing ${ }^{[2,3]}$.

Several Greek mathematicians were known for constructing automata. The first automaton was built by Archytas of Tarentum (428-347 BC), and it consisted of a pigeon-shaped steam-operated flying machine. 
Ctesibius of Alexandria (285-222 BC) was known to have built many human figures able to move and drink, as well as a black bird singing by means of a water flow. Moreover, Heron of Alexandria (10 BC-70 AD) conceived and constructed an entire theatre scene played by automata ${ }^{[3,4]}$.

In $949 \mathrm{AD}$, Liutprand of Cremona visited Constantinople and described the automata of Emperor Theophilos' palace, including metal lions striking the ground with their tails and roaring with open mouth and quivering tongue ${ }^{[5]}$.

In the Islamic world, the polymath Al-Jazari (1136-1206 AD) wrote a treatise where he described several automata he had designed and constructed, including automated musicians conceived to amuse royal guests $^{[3]}$.

During the Renaissance, several inventors were dedicated to the construction of automata, such as Giovanni Fontana (1395-1454 AD), who designed automatic war machines capable of throwing bombs, and Leonardo da Vinci (1452-1519 AD), who designed a mechanic knight able to stand up and sit down, wave its arms, and move his head and jaws ${ }^{[4]}$.

However, the real progress in the construction of modern robots was reached with the Industrial Revolution and the development of calculators, and it was surprising.

\section{From industry to surgery}

At the origin of the development of robotic technology during the 20th century, there was the concept of "telepresence", intended as the idea that people can appear, receive stimulations, and produce some effects in a place other than their real location as if they were really present. This idea animated the development of the first robotic arms intended to be used in hostile environments, such as the ocean floor, or to manipulate hazardous materials ${ }^{[3]}$.

Already in 1951, engineer Raymond Goertz designed the first teleoperated articulated arm for the United States Atomic Energy Commission to handle radioactive material safely and reduce the risks for personnel. This system was a manipulator using just pulleys and cables as mechanical coupling between operator and machine, but it already represented a major progress in terms of design and feedback technology ${ }^{[6,7]}$.

In 1954, engineer George Devol patented a programmable robotic system designed for transferring objects and conceived for a large variety of purposes. From this initial project, he developed the world's first industrial robot, Unimate. He also co-founded with engineer Joseph Engelberger the world's first robotics company, Unimation, located in Danbury, Connecticut, to produce Unimate ${ }^{[7,8]}$.

In 1961, the first Unimate robot was installed in a General Motors factory in New Jersey and consisted of a robotic arm for lifting hot metal objects from die-casting machines and stacking them. Several automobile companies soon understood the potential of this technology, and large-scale production of this robot started $^{[7,8]}$.

In 1969, Victor Scheinman, a researcher of the Stanford Artificial Intelligence Laboratory, developed the "Stanford Arm". It was an all-electric, computer-controlled, six-axis articulated robotic arm, able to follow random trajectories and perform a series of instructions, unlike previous machines, which moved along one fixed trajectory and performed only one task repeatedly. Indeed, the "Stanford Arm" was specifically designed to widen the application of robots to complex tasks, such as assembly and arc welding. Its potential applications were proved in 1974, when a sensor guided experimental version of this robotic arm managed to assemble a car water pump without any human intervention ${ }^{[7-9]}$. 
In 1977, Scheinman sold his invention to Unimation. On this basis, Unimation collaborated with General Motors and developed the Programmable Universal Manipulation Arm (PUMA), which represented the basis for the production of a series of successful industrial robots ${ }^{[7-9]}$.

With the production of PUMA, the robotic technology entered the operating theatre. Indeed, the first use of a robot in a surgical procedure was documented in 1985 by Kwoh et al ${ }^{[10]}$, who reported a CT-guided stereotactic biopsy of a brain tumor performed in a 52-year-old male patient using a Unimate PUMA 200 robotic arm at the Memorial Medical Center, Long Beach, California.

\section{Surgical robots spreading}

Since the second half of the 1980s, several robotic surgical systems started to appear in the operating theatres. In 1988, researchers from the Imperial College of London developed the PROBOT system to perform prostatic resections. In 1992, Integrated Surgical Systems, in collaboration with IBM, released the ROBODOC system, successfully used for milling the femur in hip replacement procedures ${ }^{[1,3,11-14]}$.

In the same period, a group of researchers of the National Aeronautics and Space Administration (NASA) working on virtual reality started collaborating with researchers of the Stanford Research Institute (SRI) working on accurate surgical telemanipulators for open microsurgery. After the presentation of Jacques Perrisat's laparoscopic cholecystectomy at the Society of American Gastrointestinal and Endoscopic Surgeons in 1989, the SRI developers were urged to adapt their telepresence surgical system to the new laparoscopic approach, which was immediately regarded as a perfect field of implementation for robotic technology ${ }^{[14]}$.

Meanwhile, the U.S. Defense Advanced Research Projects Agency (DARPA) started a research program to develop a robotic surgical telemanipulator mounted on a mobile armored vehicle and remotely operated by a surgeon at a rear facility area. The aim of this project was to allow surgeons to control life-threatening injuries on the battlefield and stabilize injured soldiers before they were taken away. For this purpose, DARPA funded SRI, which developed a robotic system proving successful in performing complex surgical procedures in animal models. Finally, the project was not completed for human use, but it provided a solid basis for the development of the robotic systems later used in surgery ${ }^{[14]}$.

In 1993, Computer Motion, funded by NASA and DARPA, released the Automated Endoscopic System for Optimal Positioning (AESOP), an intern replacement voice-controlled robotic arm allowing the automatic control of a camera during laparoscopic surgery ${ }^{[14]}$.

In 1996, the same company released ZEUS, a surgical system consisting of three robotic arms attached to the operating table, one of which was an AESOP, with originally six degrees of freedom (later seven) and a monitor provided console for remote control ${ }^{[14]}$. In 2001, it was used by Pr. Marescaux et al. ${ }^{[15]}$ operating in New York to perform the first transatlantic robotic cholecystectomy in a 68-year-old female patient laying on the operating table in Strasbourg, the so-called "Lindbergh Operation" ${ }^{\text {"15,16] }}$.

In 1995, Drs. Fred Moll and John Freund, together with engineer Robert Younge, founded Intuitive Surgical after negotiating for the intellectual properties of SRI robotic surgical systems. On this basis, Intuitive Surgical developed the first prototype of the da Vinci surgical system in 1997. After being ameliorated, the system received US Food and Drug Administration approval in 2000. After passing through several versions, it currently represents the most widespread and used master-slave robotic surgical system in general surgery $^{[14]}$.

It must be noted that several other robotic systems have been used in surgery thus far, but here we only select the ones appearing to mark more deeply the evolution of robotic technology in operating rooms. 


\section{Main types of robots implemented in surgery}

Some authors have distinguished the different robotic systems thus far used in surgery into three main types $^{[12]}$ :

1. "Precise path systems" include robots previously programmed to perform predefined and repetitive tasks, such as several types of devices used for prostatic transurethral resections and to puncture the renal calyces.

2. "Intern replacement systems" include robotic devices intended to replace surgical assistants in tasks requiring dexterity and stability, such as the AESOP system.

3. "Master-slave systems" have several robotic arms remotely controlled by a surgeon through a computer console, mimicking precisely on the patient laying on the operating table the movements carried out by the surgeon at the console, and never moving without the surgeon's guidance. In this context, the da Vinci surgical system has become paradigmatic.

Clearly, this is not a complete summary, but it is useful to set out some important phases in the implementation of robotic technology in surgery.

\section{MILESTONES IN ROBOTIC COLORECTAL SURGERY}

The first publications available in the literature concerning robotic colorectal surgery are reported here. A brief state of the art about the robotic surgery of colon and rectum is also provided.

\section{Robotic surgery of the colon}

The first cases of robot-assisted colectomies were published in $2002^{[17]}$. In particular, Weber et al ${ }^{[18]}$ reported one case of sigmoid colectomy for diverticular disease in a 50-year-old female patient, and one case of right hemicolectomy for cecal diverticulitis in a 43-year-old male patient. In both procedures, a da Vinci surgical system was used for large bowel mobilization, whereas colonic section and vascular ligations were accomplished with a laparoscopic-assisted technique, and anastomoses were performed extracorporeally. Moreover, the same surgical team published in the same year a comparative study reporting 15 laparoscopic colectomies performed using an AESOP 3000 robotic camera holder and 11 not robot-assisted laparoscopic colectomies $^{[19]}$.

The first cases of patients with colon cancer undergoing robot-assisted surgery with a master-slave robotic system were reported by Hashizume et al ${ }^{[20]}$ in 2002, and they consisted in one ileocecal resection, one left hemicolectomy, and one sigmoidectomy performed by means of da Vinci technology for cecal, descending colon, and sigmoid colon cancer, respectively ${ }^{[17]}$.

Since then, many studies concerning robot-assisted surgery of the colon have been published, marking a progressive amelioration of technical practices and a wide spread of competences. Among these studies, a certain attention should be paid to a case series of right and left colectomies published by Rawlings et al. ${ }^{[21]}$ in 2007, where the authors reported the first cases of robot-assisted side-to-side intracorporeal anastomosis after right colectomy.

The advantages provided by robotic systems, such as stable, immersive, and three-dimensional view; better dexterity due to seven degrees of freedom; and ambidextrous capabilities, seem to offer the potential to overcome the limitations of conventional laparoscopy, mostly due to less favorable ergonomic features. Therefore, over time, many studies were carried out to compare the outcomes of robotic and laparoscopic surgery of the colon, but their results are contrasting and clear conclusions are not possible.

In particular, a recent systematic review with meta-analysis published by $\mathrm{Ng}$ et al.$^{[22]}$ in 2009 tried to state whether robot-assisted laparoscopic surgery had better outcomes compared to conventional laparoscopy in colorectal cancer treatment. The authors included six randomized clinical trials (RCTs) and 67 prospective/ retrospective cohort studies and case-controlled studies, demonstrating that robotic surgery was superior to 
conventional laparoscopy in terms of all-cause mortality, incidence of surgical site infection, intraoperative blood loss, length of hospital stay, and time to oral diet, but inferior in terms of operative time. No significant difference was found in terms of anastomotic leak and disease recurrence. However, regarding the RCTs subgroup of the same study, no significant difference was found except for operative time, which confirms the current absence of evident advantages in favor of one approach or the other in colon cancer surgery.

\section{Robotic surgery of the rectum}

In 2001, Cadière et al ${ }^{[23]}$ first described the use of robotic technology in rectal surgery, reporting three transanal rectal resections performed by introducing through the anus two robotic arms for manipulations and a standard laparoscope held by an assistant for viewing. In 2003, Delaney at al. ${ }^{[2]}$ reported the first case of transabdominal robot-assisted rectopexy for rectal prolapse, while Giulianotti et al ${ }^{[25]}$ reported the first cases (six) of robot-assisted rectal anterior resection for rectal cancer ${ }^{[17]}$. Notably, both types of operations mentioned above were performed with Intuitive Surgical systems. In the same year, Hildebrandt et al. ${ }^{[26]}$ also reported the implementation of an AESOP 3000 robotic arm in the surgical treatment of rectal cancer to perform two laparoscopic rectal anterior resections.

The advantages provided by the robotic systems in terms of view and manipulations seemed even more evident in the case of rectal surgery because of the narrow, deep, and fixed operating field represented by the pelvis. Therefore, a growing number of robot-assisted procedures in rectal surgery, especially for cancer, has been reported, with many authors trying to compare the outcomes of robotic surgery and conventional laparoscopy.

Focusing on oncological surgery and setting aside the numerous retrospective studies available, seven $\mathrm{RCTs}^{[27-33]}$ comparing robotic and laparoscopic surgery of the rectum were carried out to present, and their results were summarized by Liao et al ${ }^{[34]}$ in a systematic review with meta-analysis published in 2019. Notably, these authors did not find any significant difference in terms of circumferential resection margins and quality of mesorectal excision, as well as in terms of proximal resection margins and number of retrieved lymph nodes, even if a significant heterogeneity of data was found for these two latter issues. On the contrary, distal resection margins were significantly longer in patients undergoing robotic surgery, although the heterogeneity of data was still considered high.

Particular attention must be paid to the Robotic vs. Laparoscopic Resection for Rectal Cancer (ROLARR) study $^{[31]}$, the largest and highest quality trial currently available. In particular, it concluded there was no significant difference between robotic and conventional laparoscopic surgery in terms of conversion rate, circumferential resection margins, mesorectal resection quality, and postoperative complications within 30 days and 6 months after operation. In addition, the authors performed a costs analysis showing significantly higher costs for robotic surgery, but the absolute difference was just slightly in favor of conventional laparoscopy among the patients with complete data.

Moreover, among the trials cited above, only Patriti et al. ${ }^{[28]}$ reported overall survival and disease-free survival, showing no significant differences, but stressing a trend towards better disease-free survival in robotic surgery and concluding in favor of the latter in the case of total mesorectal excision but not in case of partial mesorectal excision.

\section{Limitations of robotic technology to present}

Even if robotic technology provides several objective advantages in terms of working conditions and offers a faster adaptation compared to conventional laparoscopy, it also presents some limitations, the most relevant 
of which are represented by time of robotic setting, lack of both tactile sensation and tension feedback, and high $\operatorname{costs}^{[35]}$.

Robot docking and collisions among instruments partially explain the longer operating time frequently reported for robotic surgery, but an experienced team may overcome this limitation, as can the implementation of the latest technological innovations. The latter might also allow overcoming the absence of tactile feedback of robotic systems, currently partially arranged by means of the ameliorated view they provide $^{[35]}$.

For what concerns high costs, it can to be remarked that the implementation of conventional laparoscopy was also very expensive at the beginning, but it provided a number of advantages in terms of postoperative outcomes, which justified its spread. Clearly, the implementation of robotic surgery is more expensive, but the evaluation of eventual clear benefits for patients and surgeons could motivate its use as well ${ }^{[35]}$.

\section{CONCLUSION}

The implementation of robotic technology in general surgery represented the inevitable outlet of an astonishing technological development. It has proved to be feasible and safe, with several operating advantages for surgeons. However, clear advantages in terms of patient outcomes have not yet been demonstrated after the implementation of robotic systems in colorectal surgery.

To evaluate the overall impact of robotic technology in colorectal surgery, further studies with high levels of evidence are necessary, as well as those implementing the new robotic technologies already appearing in operating theatres.

\section{DECLARATIONS}

\section{Authors' contributions}

Conception: Genova P, Pantuso G, de'Angelis N

Literature review: Genova P, Pantuso G, Abdalla S, Memeo R, Gaiani F, Gavriilidis P, de'Angelis N

Writing: Genova P (Genova P and Gavriilidis P for the chapter "Ancient times: automata")

Revision: Genova P, Pantuso G, Abdalla S, Memeo R, Gaiani F, Gavriilidis P, de’Angelis N

\section{Availability of data and materials}

Not applicable.

\section{Financial support and sponsorship}

None.

\section{Conflicts of interest}

All authors declared that there are no conflicts of interest.

\section{Ethical approval and consent to participate}

Not applicable.

\section{Consent for publication}

Not applicable.

\section{Copyright}

(C) The Author(s) 2020. 


\section{REFERENCES}

1. Saffary R, Macario A, Kadry B. Historical overview of robot-assisted surgery. Chapter 2017; doi: 10.1017/9781316534229.003.

2. Needham J, Ronan CA. The Shorter Science and Civilization in China. Technology \& Culture 1980; doi: 10.2307/3104800.

3. Yates DR, Vaessen C, Roupret M. From Leonardo to da Vinci: the history of robot-assisted surgery in urology. BJU Int 2011;108:170813; discussion 1714.

4. Gasparetto A. Robots in history: legends and prototypes from ancient times to the industrial revolution. Explorations in the History of Machines and Mechanisms. Springer; 2016. pp. 39-49.

5. Safran L. Heaven on earth: art and the Church in Byzantium. Pennsylvania State University Press University Park; 1998.

6. Rosen J, Hannaford B, Satava RM. Surgical robotics: systems applications and visions. Springer Science \& Business Media; 2011.

7. Robotics timeline. Available from: https://nieonline.com/tbtimes/downloads/supplements/robotics_timeline.pdf. [Last accessed on 16 Dec 2019]

8. Munson GE. The rise and fall of unimation inc. A story of robotics innovation \& triumph that changed the world. http://www.botmag. com/the-rise-and-fall-of-unimation-inc-story-of-robotics-innovation-triumph-that-changed-the-world/. [Last accessed on 16 Dec 2019]

9. Victor Scheinman, robotics pioneer - obituary. In. The Telegraph; 26/09/2016. Available from https://www.telegraph.co.uk/ obituaries/2016/09/26/victor-scheinman-robotics-pioneer--obituary/ [Last accessed on 27 Dec 2019]

10. Kwoh YS, Hou J, Jonckheere EA, Hayati S. A robot with improved absolute positioning accuracy for CT guided stereotactic brain surgery. IEEE Trans Biomed Eng 1988;35:153-60.

11. Nguyen MM, Das S. The evolution of robotic urologic surgery. Urol Clin North Am 2004;31:653-8.

12. Otero JR, Paparel P, Atreya D, Touijer K, Guillonneau B. History, evolution and application of robotic surgery in urology. Arch Esp Urol 2007;60:335.

13. Shah J, Vyas A, Vyas D. The history of robotics in surgical specialties. Am J Robot Surg 2014;1:12-20.

14. George EI, Brand CT, LaPorta A, Marescaux J, Satava RM. Origins of robotic surgery: from skepticism to standard of care. JSLS 2018;22:e2018.00039.

15. Marescaux J, Leroy J, Gagner M, Rubino F, Mutter D, et al. Transatlantic robot-assisted telesurgery. Nature 2001;413:379.

16. Marescaux J, Leroy J, Rubino F, Smith M, Vix M, et al. Transcontinental robot-assisted remote telesurgery: feasibility and potential applications. Ann Surg 2002;235:487-92.

17. Sivathondan PC, Jayne DG. The role of robotics in colorectal surgery. Ann R Coll Surg Engl 2018;100:42-53.

18. Weber PA, Merola S, Wasielewski A, Ballantyne GH. Telerobotic-assisted laparoscopic right and sigmoid colectomies for benign disease. Dis Colon Rectum 2002;45:1689-96.

19. Merola S, Weber P, Wasielewski A, Ballantyne GH. Comparison of laparoscopic colectomy with and without the aid of a robotic camera holder. Surg Laparosc Endosc Percutan Tech 2002;12:46-51.

20. Hashizume M, Shimada M, Tomikawa M, Ikeda Y, Takahashi I, et al. Early experiences of endoscopic procedures in general surgery assisted by a computer-enhanced surgical system. Surg Endosc 2002;16:1187-91.

21. Rawlings A, Woodland J, Vegunta R, Crawford D. Robotic versus laparoscopic colectomy. Surg Endosc 2007;21:1701-8.

22. Ng KT, Tsia AKV, Chong VYL. Robotic versus conventional laparoscopic surgery for colorectal cancer: a systematic review and metaanalysis with trial sequential analysis. World J Surg 2019;43:1146-61.

23. Cadière GB, Himpens J, Germay O, Izizaw R, Degueldre M, et al. Feasibility of robotic laparoscopic surgery: 146 cases. World J Surg 2001;25:1467-77.

24. Delaney CP, Lynch AC, Senagore AJ, Fazio VW. Comparison of robotically performed and traditional laparoscopic colorectal surgery. Dis Colon Rectum 2003;46:1633-9.

25. Giulianotti PC, Coratti A, Angelini M, Sbrana F, Cecconi S, et al. Robotics in general surgery: personal experience in a large community hospital. Arch Surg 2003;138:777-84.

26. Hildebrandt U, Plusczyk T, Kessler K, Menger MD. Single-surgeon surgery in laparoscopic colonic resection. Dis Colon Rectum 2003;46:1640-5.

27. Baik S, Ko Y, Kang C, Lee W, Kim N, et al. Robotic tumor-specific mesorectal excison of rectal cancer: short-term outcome of a pilot randomized trial. Surg Endosc 2008;22:1601-8.

28. Patriti A, Ceccarelli G, Bartoli A, Spaziani A, Biancafarina A, et al. Short-and medium-term outcome of robot-assisted and traditional laparoscopic rectal resection. JSLS 2009;13:176.

29. Jiménez Rodríguez RM, Díaz Pavón JM, de La Portilla de Juan F, Prendes Sillero E, Hisnard Cadet Dussort JM, et al. Prospective randomised study: robotic-assisted versus conventional laparoscopic surgery in colorectal cancer resection. Cir Esp 2011;89:432-8.

30. Wang Y, Zhao GH, Yang H, Lin J. A pooled analysis of robotic versus laparoscopic surgery for total mesorectal excision for rectal cancer. Surg Laparosc Endosc Percutan Tech 2016;26:259-64.

31. Jayne D, Pigazzi A, Marshall H, Croft J, Corrigan N, et al. Effect of robotic-assisted vs conventional laparoscopic surgery on risk of conversion to open laparotomy among patients undergoing resection for rectal cancer: the ROLARR randomized clinical trial. JAMA 2017;318:1569-80.

32. Debakey Y, Zaghloul A, Farag A, Mahmoud A, Elattar I. Robotic-assisted versus conventional laparoscopic approach for rectal cancer surgery, First Egyptian Academic Center Experience, RCT. Minim Invasive Surg 2018;2018:5836562.

33. Kim MJ, Park SC, Park JW, Chang HJ, Kim DY, et al. Robot-assisted versus laparoscopic surgery for rectal cancer. Ann Surg 2018;267:243-51.

34. Liao G, Zhao Z, Deng H, Li X. Comparison of pathological outcomes between robotic rectal cancer surgery and laparoscopic rectal cancer surgery: a meta-analysis based on seven randomized controlled trials. Int J Med Robot 2019;15:e2027.

35. Bandar M, Sabilah J, Kim N. The current scope of robotic surgery in colorectal cancer. Adv Robot Autom 2015;2:2. 